\title{
The Arkansas Galaxy Evolution Survey: Supermassive Black Holes in the Universe
}

\author{
M. S. Seigar ${ }^{1}$, D. Kennefick ${ }^{2}$, J. Kennefick ${ }^{2}$, C. H. S. Lacy ${ }^{2}$, \\ J. C. Berrier ${ }^{2}$, P. Treuthardt ${ }^{1}$, I. A. Al-Baidhany ${ }^{1}$, R. S. Barrows ${ }^{2}$, \\ B. Davis ${ }^{2}$, J. A. Hughes ${ }^{2}$, A. Schilling ${ }^{2}$, D. W. Shields ${ }^{2}$, \\ and A. D. Sierra ${ }^{1}$ \\ ${ }^{1}$ Dept. Physics \& Astronomy, University of Arkansas at Little Rock \\ ${ }^{2}$ Dept. Physics, University of Arkansas at Fayetteville
}

Keywords. galaxies: fundamental parameters, galaxies: nuclei

Work done to date on the SMBH mass function has brought to light some interesting puzzles, the best known of which is cosmic downsizing. The solution to this problem is based upon the methodology by which we determine SMBH masses, which comes mainly from studies of active galaxies. The most massive SMBHs cease to be active at earlier epochs than their less massive counterparts, thus leading to an apparent decrease in the SMBH mass function with time. The larger SMBHs should still be present in the local Universe, but they lie in quiescent galaxies. This lack of certainty reflects the biggest gap in the observational record - the mass of SMBHs in quiescent galaxies. We are addressing this issue, using three methods described here:

Spiral arm pitch angle: We are exploiting a relationship between the tightness of the spiral arms of local galaxies (measured using the pitch angle) and the mass of their central SMBH as determined by Seigar et al. (2008; see Figure 1) to determine the masses of SMBHs out to $z \approx 1$ using archival HST imaging data.

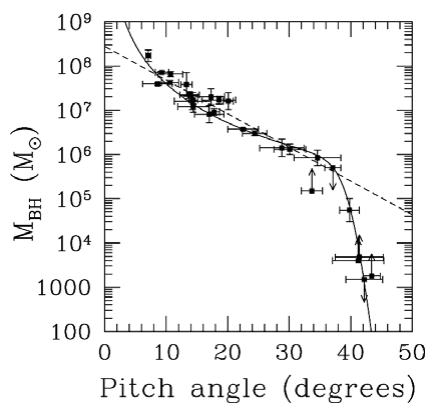

Figure 1. The correlation between the SMBH mass and the pitch angle of spiral arms.

Binary SMBHs: A very tantalizing source for LISA will be binary SMBH mergers. We are searching for SMBH binaries using archival Chandra images of ULIRGs.

Quasar Gas Dynamics: We are estimating SMBH masses for a sample of SDSS quasars at $z=1.88,2.80$, and 4.06. To compute the SMBH mass, we are using single-epoch spectra to measure the speed of gas in the broad-line region (BLR) by measuring the linewidths. To probe possible evolution in the relation in Figure 1, we are using a sample of nearby spirals from the GOODS fields that elso exhibit AGN activity, and we can then use both spiral arm pitch angle and BLR dynamics to estimate the SMBH masses.

\section{Reference}

Seigar, M. S., Kennefick, D., Kennefick, J., \& Lacy, C. H. S. 2008, ApJ, 678, L93 